\title{
ㄷำ1
}

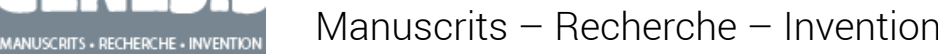

46 | 2018

Entre les langues

\section{Luba Jurgenson - Écrire entre deux corps}

\section{Julia Holter}

\section{OpenEdition}

\section{Journals}

Édition électronique

URL : http://journals.openedition.org/genesis/3029

DOI : 10.4000/genesis.3029

ISSN : 2268-1590

\section{Éditeur :}

Presses universitaires de Paris Sorbonne (PUPS), Société internationale de génétique artistique littéraire et scientifique (SIGALES)

\section{Édition imprimée}

Date de publication : 4 juin 2018

Pagination : 121-129

ISBN : 979-10-231-0604-6

ISSN : $1167-5101$

\section{Référence électronique}

Julia Holter, «Luba Jurgenson - Écrire entre deux corps », Genesis [En ligne], 46 | 2018, mis en ligne le 01 juin 2019, consulté le 07 septembre 2019. URL : http://journals.openedition.org/genesis/3029 ; DOI : 10.4000/genesis.3029 


\title{
Écrire entre deux corps Entretien avec Luba Jurgenson
}

\author{
Propos recueillis par Julia Holter
}

Luba Jurgenson, née en 1958 à Moscou, vit à Paris depuis 1975. Elle a publié son premier livre (Avoir sommeil, recueil de nouvelles, Gallimard) en 1981. Elle est auteur de romans, dont Éducation nocturne (Albin Michel, 1994), Boutique de vie (Actes Sud, 2002), Trois Contes allemands (Pierre Guillaume de Roux, 2012) et d'un essai Au lieu du péril (Verdier, 2014) consacré aux questions de bilinguisme. Traductrice entre autres de Nina Berberova, Varlam Chalamov, Marina Tsvetaïeva, Boris Pasternak, elle enseigne la littérature russe à l'université de Paris IV et mène un travail de recherche sur le témoignage littéraire et les représentations des violences de masse.

Julia Holter - Pour vous, traductrice et écrivaine française d'origine russe, "une noga [n'est] jamais tout à fait la même chose qu'une jambe, ne serait-ce que parce qu'elle est à la fois jambe et pied. [...] Une main n'est pas tout à fait la même chose qu'une rouka, qui est à la fois main et bras.» Et vous ajoutez : "Donner la parole au bilinguisme : lui faire raconter ce que vivre entre deux langues fait au corps. [...] On a tous les membres, tous les organes en double. On a une tête et une golova, deux jambes et deux nogui, un cour et un serdtse ${ }^{1}$. » L'impossibilité du «mot-à-mot» corporel correspond pour vous à une inadéquation d'un corps à l'autre là où nous aimerions que le corpus (textuel) et corps (physique) ne fassent qu'un. Ainsi, votre corps russe correspond à la période de l'enfance. C'est un corps intime, corps «maison», dites-vous. Pourriez-vous revenir sur ce corps et sa langue "figée » depuis l'âge de 17 ans, l'âge qui était le vôtre quand vous avez quitté l'URSS?

Luba Jurgenson - Au temps de mon enfance soviétique, il y avait une série de blagues dont le personnage principal était Tchapaïev, un héros de la guerre civile devenu une sorte de mythe. Dans une de ces blagues, on voit des écoliers visiter le musée Tchapaïev. Ils contemplent les habits de Tchapaïev, l'arme de Tchapaïev, enfin le squelette de Tchapaïev. Dans un coin de la salle, un des écoliers remarque un autre squelette, tout petit. Il demande : et ça, c'est quoi? Et le guide de répondre : «C'est le squelette de Tchapaïev enfant.» Je trouve que cette blague en dit long sur la mémoire collective, mais aussi sur la mémoire individuelle. Dans mon musée linguistique, il y a une pléiade de squelettes d'âges différents. Ils cohabitent et interagissent. Or le squelette, c'est ce qui nous fait tenir. Le musée, c'est aussi la mise en scène d'une mythologie. L'enfance nourrit les réécritures de soi et le corps d'enfant enfoui n'est pas complètement figé, il est sans cesse revisité avec des yeux nouveaux, les yeux de l'adulte qui avance en âge. Il y a, certes, ce phénomène que les émigrés ont observé : une part d'eux-mêmes demeure inchangée, elle se cristallise à l'âge qu'ils avaient lorsqu'ils ont quitté leur pays. C'est un peu comme la prison (toutes proportions gardées) : on en sort à l'âge où l'on y est entré. La personne se développe normalement, peut être tout à fait adulte dans ses relations et sa vie professionnelle, mais le squelette de l'enfant se tient toujours dans un coin. Quand on a changé de langue, ce squelette de la langue d'origine demande à être entendu et parlé. Cela ne veut pas dire que je m'exprime dans un russe «infantile», mais ma langue russe est comme une coprésence de mon être de 17 ans. Bien sûr, elle a évolué, elle s'est nourrie de littérature et de critique littéraire, j'écris des articles en russe. Mais elle ne s'est pas enrichie au jour le jour d'expériences intimes, elle n'a pas été travaillée, métabolisée par le corps, elle n'a pas reçu de corps adulte. Lorsque j'utilise le matériau de l'enfance dans mes livres - et je le fais souvent -, c'est une démarche de l'ordre de l'archéologie, une archéologie de soi-même, qui

1. Luba Jurgenson, Au lieu du péril. Récit d'une vie entre deux langues, Paris, Verdier, coll. « Jaune », 2014, p. 13. 
offre des découvertes peut-être autrement plus étonnantes que si j'étais restée dans la continuité linguistique par rapport à ce corps d'enfant.

J.H. - Vous publiez votre premier recueil de nouvelles Avoir sommeil directement en français, même si, en 1981, vous considérez le français encore comme une langue étrangère. Aujourd'hui, huit livres plus tard, le français est devenu en quelque sorte votre langue-sujet, langue opérationnelle et professionnelle, et le russe langue-objet (de vos études et observations). Existe-t-il, à un moment donné, une interaction, une cohabitation entre ces deux langues? Vous arrive-t-il parfois d'écrire en deux langues à la fois ?

L.J. - Aujourd'hui, il m'est difficile de dire à quel moment le français est devenu ma langue «natale», celle d'une seconde naissance. Je pense à un recueil de Pasternak qui s'intitule Seconde Naissance. Il existe une vaste littérature critique autour de ce recueil et de ce titre. Moi, je me dis que la «seconde naissance» est la naissance dans une nouvelle langue - et je me saisis de cette formule pour désigner le changement de langue dans ma propre histoire. Car le «moi» se targue d'être un «original», je pense même que cela peut être une définition du moi, l'illusion d'être un original. On ne devient pas une traduction de soi-même, on renaît dans une autre langue. Évidemment, le bilinguisme conduit à relativiser ces notions d'original et de copie et, par conséquent, fait prendre conscience d'un moi pluriel. Avec chaque livre, j'ai l'impression de changer de langue. Lorsque je commence un nouveau texte, je ne peux plus lire les précédents, je ne les comprends tout simplement pas. Pourtant, il y a une continuité et même des retours obsessifs aux mêmes thèmes, aux mêmes images, mais c'est comme si cette continuité se déroulait de l'autre côté du miroir. On pourrait rabattre ce phénomène sur le concept de la mort de l'auteur. Mais l'auteur n'est pas mort, il existe toujours, dans une autre langue. Peut-être que je le vis ainsi parce que, précisément, j'ai expérimenté une fois ce «passage». Dans la vie, ce n'est pas du tout aussi brutal qu'avec les livres, il n'y a pas eu de coupure précise, pas de moment où j'ai pu me dire : tiens, la langue russe est maintenant une langue de derrière le miroir. Progressivement, j'ai senti qu'en français, mes mots, je les «avais dans la peau », comme dit l'expression populaire, parce que d'une certaine manière, j'avais changé de peau. Cela n'empêche pas une création en russe aussi : de petits textes qui viennent spontanément, la plupart du temps des histoires très brèves ou des aphorismes, des poèmes, des fragments de rêves qui se présentent en russe; je les note, en général ils sont très structurés, parfaitement achevés, il n'y a plus qu'à les écrire.

À part les cas célèbres de bilinguisme littéraire comme chez Nabokov ou Beckett, ou Paul Celan, il y a une autre forme de bilinguisme qui m'intéresse tout autant : celui d'un Hoffmann qui écrit de la musique et des récits fantastiques, celui d'un William Blake ou d'un Serge Prokofiev, d'un Bruno Schulz ou d'un Henri Michaux, ce sont des cas fréquents. Parfois, nous ne connaissons l'œuvre de ces créateurs que dans une langue, ou bien nous privilégions l'une de leurs langues, parce que dans l'autre ils ne se sont pas réalisés au même niveau; il s'agit pour eux d'une langue mineure, moins bien maitrisée, ou parce qu'ils y ont renoncé. Parfois, on peut se poser la question : pourquoi le génial Hoffmann a-t-il eu besoin d'écrire ses opéras, qui sont tellement en dessous de ses contes (mais en était-il conscient à l'époque ?), pourquoi le génial Prokofiev a-t-il écrit des récits médiocres (comparés à sa musique)? Je me dis qu'ils avaient besoin d'expérimenter le bilinguisme, le changement de langue, de quitter parfois une langue, voire la trahir, pour faire don de ce renoncement à l'autre langue, celle qui allait les accueillir pleinement. Il y a peut-être là quelque chose de l'ordre de l'offrande sacrificielle : le prix à payer pour se réaliser dans une langue. Pasternak a abandonné la musique alors qu'il était au seuil de la réussite, il a aussi abandonné la philosophie après avoir fait des études assez poussées. Je crois, j' ai envie de croire qu'il avait besoin d'arriver à ce tournant pour faire son choix, qu'il avait besoin de maîtriser le langage de la musique afin d'y renoncer, sans cela le changement de langue n'aurait pas eu la même valeur. C'était le prix de la seconde naissance.

D'ailleurs, on parle de «grandes langues », le russe, le français, ou encore la littérature, la musique. Mais au sein de ces vastes territoires, il y a bien sûr des multitudes de petites langues, par exemple la langue de la prose des années 1920, ou la langue des Juifs piémontais (Primo Levi), ou la langue des jeunes de tel lycée de Paris, que sais-je. On peut être plurilingue sans sortir de sa langue maternelle.

J'ai donc découvert cette possibilité de plurilinguisme au sein d'une même communauté linguistique et d'un seul livre.

Sur le chemin de l'appropriation d'une langue, il y a eu une phase de rencontre et de reconnaissance avec les mots. 
Cette phase où l'on se présente à eux et ils se présentent à nous. On imagine alors le moine d'après l'habit, je veux dire le sens d'après la sonorité, c'est une phase un peu régressive vers la pensée « magique», vers un signe non arbitraire. Par exemple, dans mon recueil de 1981, une nouvelle commence par la phrase : «Je dormais d'un sommeil astringent et concave.» Cela n'a aucun sens. À l'époque, je ne savais pas ce que voulaient dire ces deux mots, simplement ils me plaisaient. J'ai donc fait ce pari : les intégrer à mon texte, avec le risque d'écrire une absurdité. Roger Grenier, mon éditeur chez Gallimard, l'avait accepté. Cela ne l'avait pas gêné. C'était une trace de mon passage à une autre langue, une trace de ce rapport au français langue «étrangère ». Il fallait qu'il y ait des mots incompréhensibles pour moi et potentiellement opaques pour le lecteur. Après les avoir cherchés dans le dictionnaire, j' ai eu un peu honte de l'allure absurde de cette phrase, mais je les ai laissés. Aujourd'hui, je me dis que ce n'était pas si absurde. Dans «astringent» on entend stringere, étreindre, astreindre, on entend la contrainte, et aussi stranger, l'étranger. C'était une contrainte que je m'étais donnée pour inviter l'étranger dans mon texte.

J.H. - Quand vous écrivez entre vos deux corps, que se passe$t$-il au juste? Votre corps français, extime, professionnel et adulte, ne cherche-t-il pas à couvrir et à protéger (mais aussi un peu découvrir et retrouver) l'autre corps, plus fragile et lointain?

L.J. - J'ai suggéré tout à l'heure que la coexistence des «squelettes» de différents âges n'était possible que sur un fond «mythologique». La réécriture de soi repose sur la création de mythes personnels et familiaux. Je me sers beaucoup de matériau autobiographique, ce qui suppose la mise en scène et le décryptage de micro-événements, y compris verbaux : tel mot qui nous accompagne et fait sens dans notre vie en dévoilant ses facettes plurielles. Cela revient à se réinventer avec chaque livre, à projeter vers le passé, vers l'enfance une histoire, des récits qui façonnent ce corps d'enfant a posteriori. Cette démarche participe des anachronismes que l'on construit pour avancer. Il m'arrive de me demander ce que je serais devenue si j'avais émigré vers un autre pays que la France. C'était plus que probable, car l'émigration juive des années 1960-1980 se dirigeait essentiellement vers les États-Unis et le Canada. La France n'accueillait pas les Juifs soviétiques, je suis donc une exception. Ce miracle a eu lieu grâce à la famille que nous avions à Paris, mais cela aurait pu ne pas se faire. Je ne sais rien de ces doubles qui, dans un monde virtuel, vivent et écrivent en d'autres langues. Je ne sais rien non plus de celle qui serait restée en URSS. C'est un corps astral qui se tient silencieusement à la lisière de ma conscience. Lorsque je voyage, il me rend parfois visite, souvent sous forme de poème en russe. Lorsque j'étais aux États-Unis la dernière fois, $\mathrm{j}$ ' ai imaginé celle qui vivrait aux antipodes et marcherait la tête en bas. C'est l'image qui m'est venue : moi et mon double, représentés à la manière de figures de carte, tête-bêche.

J.H. - La maîtrise d'une autre langue ne fait que formaliser sa nature dialogique double. Le métier du traducteur me semble en ce sens très similaire à celui d'écrivain. Ces deux métiers que vous exercez parallèlement-ne deviennent-ils pas une seule et même chose, dans la mesure où les deux permettent de prolonger et d'élargir l'expérience du passeur entre vos deux corps, pour autant que chaque "passage» vous fait revivre votre double identité et vous conduit, la narrant, à la remanier?

L.J. - Lorsque le projet de faire des traductions est né, ce n'était pas tant pour ma réalisation personnelle que pour faire connaître en France la littérature de l'underground soviétique. Je voulais donner la parole à ces écrivains qui ne l'avaient pas dans leur pays. Mais le temps de me former en traduction, d'accéder à l'espace éditorial (grâce à Jacques Catteau), cette littérature avait été en grande partie traduite. J'ai donc commencé par des classiques, Oblomov de Gontcharov, Saltykov-Chtchedrine. Cela a été une école formidable, mais loin de mon projet initial : cet autre qu'il s'agissait de faire exister en français était bien différent de celui que j'avais imaginé. Par la suite, j'ai traduit des textes de la littérature contemporaine qui me sont proches, les romans de Leonid Guirchovitch, de Sergueï Lebedev. J'ai tendance à séparer l'expérience de la traduction de celle de l'écriture. En traduisant, je crée du texte, mais je ne crée pas le texte, je ne crée pas le Projet, je demeure dans l'aura d'autrui (au sens benjaminien) et contribue, dans l'idéal, à son accomplissement. Une des façons de créer de l'unité entre les deux activités, c'est de me dire qu'un texte n'est jamais 
terminé, même dans la langue de l'original. En témoignent les lectures que nous faisons des textes du passé, ces lectures sont différentes de celles des contemporains, nous continuons en tant que lecteurs à écrire ces textes. Le traducteur est un super-lecteur, il partage sa lecture et permet aux lecteurs de la langue cible de continuer à écrire le texte. En un sens, comme le texte n'appartient pas à son auteur mais à la langue, à la culture, on peut dire en effet qu'écrire et traduire est la même chose : mes textes ne m'appartiennent pas non plus en propre, je ne sais pas ce que la langue, à travers ses lecteurs, décidera d'en faire. Mais mon texte émane d'un projet qui est le mien, je préfère finalement le terme «projet» à celui d'aura, moins mystique, plus artisanal. On pourrait aussi parler de facture, au sens des modernistes russes. Ou encore, on peut revenir à l'idée de squelette : l'auteur crée l'ossature du texte, sa syntaxe profonde, le traducteur intervient au niveau de la morphologie, il lui invente des membres. Je peux m'approprier la partie émergée de l'œuvre d'autrui, pas ses soubassements. Reste que l'acte de traduire est magique en soi, je suis toujours émerveillée d'observer cette alchimie, la transformation d'une réalité vécue dans une langue vers une autre langue, émerveillée de constater qu'il existe des équivalents, même si on peine à les trouver. Ils se situent au-delà de la valeur de dictionnaire des mots. Il y a le dictionnaire que l'on porte en soi, c'est la première phase, technique. On le quitte pour aller vers l'encyclopédie, et l'encyclopédie n'est pas en moi, elle est à l'extérieur, c'est la culture. Le métier de traducteur est très solitaire, mais grâce à cette dimension d'encyclopédie, le monde entre en vous. Le dictionnaire, c'est la première étape. Prenons n'importe quel mot. Par exemple le mot son en russe. Pour le traduire, on a deux mots en français : sommeil et rêve. On dit en français «j' ai fait un rêve». J' aimerais bien «faire des rêves », mais en réalité, la plupart du temps, nous ne faisons pas nos rêves, car «faire quelque chose» suppose une action consciente. En russe, les rêves nous arrivent, on pourrait même dire qu'ils nous «arrêvent», mne prisnilsia son. Lorsque je traduis cette phrase par «j' ai fait un rêve», je trahis déjà le sacro-saint original. En revanche, il y a un mot en russe pour le rêve éveillé : metchta. L'ambiguïté n'est pas possible entre ces deux sens, alors qu'elle l'est en français, je peux laisser planer un doute. Ces tournures passives ou ces ambiguïtés qui ne sont pas transmissibles au niveau du dictionnaire sont des sources d'infinis va-et-vient entre les langues et d'une sorte de grand texte russo-français dans lequel je vis. On peut passer sa vie à retourner les mots, et c'est ce que je fais. Je peux aussi partir de la transcription du mot russe en français, son et imaginer un jeu sur les sonorités des mots dans les rêves. Dans une traduction, la plupart du temps, ce jeu mental n'est pas visible, on se contentera de «j' ai fait un rêve» comme équivalent de mne prisnilsia son, car c'est ce que les gens disent dans leur vie quotidienne. Mais si le rêve a vraiment une importance dans le texte, je chercherai d'autres mots. Là où la traduction rejoint l'écriture, c'est que, naturellement, je vis avec ces deux façons de dire les choses. Je dis «j' ai fait un rêve», mais derrière il y a l'autre formule, celle où le rêve advient, une formule plus archaïque parce que plus ancienne dans mon univers et aussi dans notre culture (le rêve comme dialogue avec une autre réalité ou avec les ancêtres et non comme langage de l'inconscient). Les deux expressions coexistent dans ma tête, et cette traduction perpétuelle qui s'exerce en moi parfois à mon insu constitue un laboratoire et un réservoir d'images pour décrypter le monde.

\section{J.H. - Vous avez décrit merveilleusement comment le corps «traduit» entre deux rives :}

Le passage physique d'une langue à l'autre dans mon corps. Cela se fait en deux temps. D'abord une lecture bilingue. Je laisse le texte me traverser, aller d'une rive à l'autre, vivre simplement en moi en deux langues à l'instar des objets du monde, je le lis comme ma propre vie. [...] À ce stade du texte, je ne le vois pas, je suis à l'intérieur, au plus près de la situation du passage, dans ce passage. Si à ce moment-là on me soumettait à un examen aux rayons $\mathrm{X}$, on verrait les mots bouger et se métamorphoser. De temps en temps, mon œil intérieur les saisit : un tel, dont les pattes de devant et le museau sont déjà français, traîne encore sa queue en russe ${ }^{2}$.

Après cette «lecture " métamorphosante arrive la deuxième phase, celle qui consiste à rendre, dans la langue cible, l'étrangeté, l'originalité du texte. Vous ne regardez. plus le texte russe, vous en fermez la porte : "Je lui crée une nouvelle genèse 3 . »

2. Luba Jurgenson, Au lieu du péril. Récit d'une vie entre deux langues, op. cit., p. 79

3. Ibid., p. 80 . 
Mais que se passe-t-il quand vous vous mettez à écrire votre propre livre? Comment le corps bilingue mène-t-il son dialogue intérieur? Le corps russe, son intimité et son gosier, comment fait-il irruption dans le processus créatif?

L.J. - Je vais parler du livre que je suis en train d'écrire, car cela m'est plus facile. C'est, en un sens, un livre plurilingue. J'y donne une place à ce corps russe. Il y a là des passages en russe, quelques passages en d'autres langues aussi. Il est composé d'histoires structurées et de fragments, et ces fragments me viennent parfois en russe, je les prends tels quels. Jusqu'à il y a quelques jours, je ne savais pas trop comment articuler les deux langues, comment présenter la traduction de ces passages. Puis, j'ai compris que les passages en russe - incompréhensibles pour le lecteur français et donc, donnés aussi en traduction à un autre endroit - viendront s'insérer précisément là où quelque chose d'incompréhensible intervient dans la narration. Ils figurent ces opacités du sens, ces barrages qui entravent l'œuvre d'interprétation du monde menée au cours de notre vie. Ils apparaissent donc à la place des non-dits ou lorsque je choisis une posture narrative impossible - là où mon narrateur est à la fois lui-même et quelqu'un d'autre ou lorsqu'il se rend compte qu'il est mort, etc., ou lorsqu'il est question de paroles entendues dans l'enfance et qui n'ont pas été comprises sur le moment, mais seulement des années plus tard. Si un jour ce livre est traduit en russe, il faudra faire l'inverse, insérer du français à ces endroits. C'est en quelque sorte un livre réversible. Donc, la traduction fait partie de l'écriture. J'ai déjà pensé écrire un livre où une même histoire serait traduite plusieurs fois du français en russe, puis du russe en français, jusqu'à ce qu'il n'en reste plus rien, comme lorsqu' on change plusieurs fois un billet de banque - d'euros en dollars, de dollars en livres, de livres en couronnes etc., à la fin il ne reste plus rien. Cela devient un «billet de manque». C'est d'ailleurs drôle que l'on parle de «conversion» à propos d'argent. La traduction suppose une conversion, celle à une nouvelle croyance, la croyance dans les mots de la langue. Je ferai peut-être un jour cette expérience, pour mettre par écrit ce manque, mais aussi ce surplus qu'apporte la traduction. J'ai aussi envisagé une supercherie littéraire qui consisterait à présenter un de mes livres comme une traduction. J'ai été tentée de le faire à une époque où la littérature russe était à la mode, pour attirer l'attention. Mais cela n'a pas été possible, car je n'écris pas de littérature russe. Mes lecteurs russes me disent que ce que j'écris n'est pas russe du tout. Dans mon roman précédent, Trois Contes allemands, il y a trois histoires présentées comme traduites de l'allemand. Il est écrit «roman» sur la couverture, je n'ai donc pas trompé le lecteur, mais certains se sont pris au piège et cela m'a enchantée. J'ai tenté dans ce livre une expérience plurilingue en français, dans un français qui se veut traduction de l'allemand. Parce que l'allemand est venu progressivement désigner ce qui est incompréhensible, l'Allemagne est un territoire métaphorique, le lieu de l'unheimlich, inquiétante étrangeté, que Freud ne confronte d'ailleurs pas tant à heimlich, secret, qu'à heimisch, le familier, lui-même dérivé de Heimat, la patrie. Donc, l'Allemagne figure le déplacement, le détour, ce détour qu'il faut accomplir pour que soient dites les choses qui ne peuvent l'être directement, c'est la patrie des mots apatrides. Un lieu où l'on peut expérimenter la conversion du «manque» en surplus. Mon «roman» avec l'Allemagne a commencé à partir d'Éducation nocturne (1994), mais à l'époque, je n'avais pas encore perçu toutes les possibilités de conversion que m'offrait ce détour entre la Russie et la France. Dans Trois Contes allemands, il y a trois époques : la veille de la Grande Guerre, le lendemain de la Seconde Guerre mondiale et aujourd'hui. Donc, trois langues. L'idée, c'était de travailler le rapport de la langue à l'histoire : la langue fait l'histoire en même temps qu'elle l'écrit. J'y ai aussi expérimenté une autre forme de plurilinguisme, la forme poétique, qui permet de représenter une langue étrangère.

J.H. - Participez-vous à la traduction de vos livres? Vous autotraduisez-vous? Si oui, pourriez-vous nous parler de vos expériences de l'autotraduction et/ou de la traduction collaborative?

L.J. - J'ai co-traduit vers le russe deux de mes livres, Éducation nocturne et Trois Contes allemands, en collaboration avec Leonid Guirchovitch, un auteur de l'émigration, qui vit en Allemagne et que je traduis moi-même en français. Une sorte de mouvement spéculaire, donc. Pour répondre à votre question, il faut que j'explique comment ce travail a commencé. J'ai découvert le premier roman de Leonid Guirchovitch au moment de la Perestroïka. En manuscrit, car à l'époque il était impensable de le publier en russe. Ce roman est paru en français en 2004 sous le titre Apologie de la fuite, aux Éditions Verdier. Or il avait été écrit à une époque où l'URSS était encore bien solide, commencé dans 
les années 1970 et terminé au début des années 1980. L'auteur y imagine la chute de l'URSS (le livre ne se réduit pas à ce scénario de politique-fiction, c'est un roman de formation, c'est aussi un très grand texte sur la musique et sur la judéité). C'était une œuvre qui ne ressemblait à rien de ce que je connaissais, d'une originalité absolue, qui a bouleversé ma conception du roman. J'ai voulu la faire connaître en France. Mais la narration en était si complexe, si stratifiée, que j'ai eu besoin de travailler avec l'auteur. Leonid Guirchovitch était aussi violoniste, il jouait à l'orchestre de Hanovre. Au cours de notre travail commun, $\mathrm{j}$ ' ai appris qu'il était en train d'écrire un roman dont le héros principal était un violoniste juif; l'action se déroulait en Allemagne pendant la guerre. J'étais moi-même en train d'écrire Éducation nocturne, sur un thème très proche. Finalement, ma traduction d'Apologie de la fuite n'a abouti que douze ans plus tard. Entre-temps, l'URSS s'était bel et bien décomposée. Avec la chute de l'URSS et l'ouverture des frontières, mon identité russe émigrée avait volé en éclat. Il n'y avait plus d'émigration et je ne me reconnaissais pas du tout dans la Russie qui avait émergé. C'est à ce moment-là que j'ai vraiment «naturalisé » mon français, que le français a cessé d'être une langue étrangère. Je ne pouvais plus du tout me considérer comme russe, car désormais, cela signifiait «citoyenne de Russie». J'étais en quelque sorte une autre personne, avec d'autres références. Il a donc fallu refaire la traduction, car le monde avait changé et ma lecture de ce texte aussi. En 2004, lorsque la version française du livre était au stade des épreuves, Leonid Guirchovitch, qui n'est pas francophone, m'a demandé de traduire une page de l'un de mes romans : il voulait se faire une idée de la personne qui le traduisait. Je lui ai donné une page d'Éducation nocturne. C'était pour moi une première vraie expérience d'autotraduction. Il l'a retravaillée, et lorsque j' ai lu cette page reprise par lui, j' ai eu le sentiment qu'il avait vraiment saisi mon écriture à la source : ce que je lisais était un original. C'était comme si pendant toutes ces années j'avais écrit et existé en traduction. Une expérience tout ce qu'il y a de plus unheimlich. Il m'a proposé alors de traduire tout le livre ensemble. C'est ce que nous avons fait : je lui adressais des pages en russe et il les reprenait. D'ailleurs, à la différence de mes autres livres, j'avais l'impression qu'Éducation nocturne n'était pas terminé. J'ai dit plus haut qu'aucun livre n'était jamais achevé, mais par rapport à ce roman, ce n'était pas une position de principe, c'était une sensation très concrète. Par exemple, il m'est arrivé, alors qu'il était déjà paru, d'écrire de nouveaux passages (en russe d'ailleurs). Ces passages n'ont jamais vu le jour, ils se sont perdus dans mes nombreux cahiers. En revanche, j' ai écrit bien plus tard un chapitre qui ne figurait pas dans ce roman, pour le numéro de la revue d'Autrement dont le thème était Mères et fils. Ce travail de traduction a été initié dix ans après la parution du livre. Autant dire que je l'ai entièrement réécrit. Je l'ai revécu. Aujourd'hui, je ne sais plus où est l'original et où est la traduction. C'est le même livre et ce n'est pas le même.

Ce roman porte sur la guerre, sur la Shoah, sur la musique. Leonid Guirchovitch m'a beaucoup aidée à imaginer l'aspect physique et matériel du métier de violoniste. Mon éthique par rapport à la représentation de la Shoah avait changé aussi, sans doute au contact de mes recherches sur l'écriture de la violence extrême. Éducation nocturne était un exercice d'ironie - mais une ironie discrète. Dans la version russe, ce fond d'ironie s'est accentué. J'entends par là cette distance que l'on peut qualifier de postmoderne, une non-croyance affichée dans la réalité. Le postmodernisme est en un sens un courant très platonicien, il suggère que tout est représentation, et il s'y complaît, à la différence du réalisme qui sait également que tout est représentation, mais qui s'astreint à l'ascèse de la foi, qui fait semblant de croire à la réalité. La traduction russe est plus ouvertement postmoderne que l'original français, ou l'original russe plus postmoderne que l'original français, c'est plus juste de le dire ainsi, ou encore, la traduction russe plus postmoderne que la traduction française, car au fond, il n'y a plus d'original du tout. Dans la version russe, le monde est clairement décor. Certaines scènes vécues par les personnages sont devenues des récits rapportés ou des rêves, mais ce procédé trop facile ne me satisfaisait pas vraiment. Alors, à un moment, cela m'est venu : une scène que je voulais raconter de manière distanciée s'est présentée en vers. Des vers rimés, une sorte de parodie du romantisme. J'ai quitté la langue russe à un âge où l'on lit beaucoup de poèmes et où l'on en écrit. Mon bagage poétique d'enfance, c'est bien sûr Pouchkine, Baratynski, Alexeï Tolstoï, les romantiques allemands dans les traductions de Joukovski, Heine en traduction. Ou les livrets d'opéra. C'est ce genre de vers que j' ai utilisé. Aujourd'hui, ma mémoire est remplie de poésie moderniste russe, surtout futuriste d'ailleurs, mais le XIX ${ }^{\mathrm{e}}$ siècle reste très près du corps dès lors que j'active la 
fibre parodique. La parodie, pour moi, est quelque chose de très émotionnel, très intime. La vraie parodie, c'est quand on se moque de ce que l'on aime profondément. On met des guillemets à un texte sans lesquels l'histoire littéraire ne permet plus d'exister. Certes, avec le temps les guillemets s'effacent. Mais la langue reconnue immédiatement comme poétique (avec des rimes) et comme appartenant à une autre époque produit un effet de distanciation puissant.

J'ai ainsi expérimenté un plurilinguisme que je n'ai pas fini d'explorer. Un peu plus tard, en traduisant le roman de Guirchovitch Schubert à Kiev, je me suis trouvée devant une difficulté : il y avait des passages en ukrainien. Ces passages avaient une fonction ludique, ils étaient drôles, des implants comiques sur fond de terreur de la Seconde Guerre mondiale en Ukraine. Si je les avais simplement traduits en mettant une note, on aurait perdu une dimension essentielle du texte. Alors, j' ai trouvé : je les ai traduits en vers, en vers d'opéra, puisque l'action se déroule à l'opéra de Kiev. À partir de là, c'est devenu un procédé plus conscient.

Dans Trois Contes allemands, il y a un long poème romantique, écrit par l'un des personnages soi-disant en allemand, et par moi en français. Je me suis donné une contrainte formelle très forte. En le traduisant en russe, il a fallu modifier la prosodie. Les poètes de mon enfance ont repris le dessus, le pentamètre iambique s'est imposé.

Il y avait d'autres poèmes dans Trois Contes allemands, l'ébauche d'un opéra. Dans la version française, je m'étais inspirée de la traduction russe de Faust par Pasternak. Il s'éloigne beaucoup du texte allemand, je m'éloignais du sien en l'adaptant en français, j' arrivais donc à camoufler ma source. En traduisant ce roman vers le russe, je ne pouvais pas me référer à cette traduction que tout le monde connaît. J'ai donc dû ajouter une «couche» au millefeuille.
C'est à la suite de ce jeu que j'ai pris, dans le texte que j'écris aujourd'hui, encore plus de libertés avec les langues.

Au lieu du péril est en cours de traduction en anglais, je relis le texte, mais là je ne participe pas de manière active à sa création, je ne fais que vérifier, suggérer.

J'ai le projet de retraduire Éducation nocturne du russe en français, ce sera encore un autre livre bien sûr. J'ai commencé à le faire et je me suis arrêtée. Ce livre est le résultat d'un grand désir du romanesque qui m'a guidée au début de ma vie d'écrivain. Lorsque j'ai écrit Avoir sommeil, c'est parce que je me sentais toute petite, il fallait donc que mes textes soient petits, mais j'aspirais à grandir, à écrire un roman. D'ailleurs, en France on ne jurait que par le roman (et c'est toujours le cas), comme si on était toujours au XIXe siècle. J'ai cheminé en écrivant des textes présentés comme des romans, qui n'en étaient pas. Puis, il y a eu Le Soldat de papier et surtout Éducation nocturne, l'aboutissement de mon projet romanesque. Après quoi, ma foi dans le roman s'est effritée. J'en écris toujours, mais ce sont des romans qui interrogent le genre. Cette remise en question a coïncidé avec la rencontre de l'œuvre de Varlam Chalamov, qui est un «anti-romancier» par excellence. On ne sort pas indemne d'une telle rencontre. Éducation nocturne était en un sens un roman traditionnel, avec un héros et une histoire. Or, pour le traduire, il faudra le revivre encore une fois, c'est-à-dire, me confronter aux questions que me pose l'esthétique du roman. Parce que, entre-temps, j'ai changé de langue, quittant celle du roman pour une écriture hybride. Autant dire qu'il ne s'agit pas simplement d'une traduction du russe en français. Vivre un livre, c'est aussi une expérience physique, un peu comme une épreuve sportive, il faut donc que je sois vraiment en forme pour composer, dans une autre langue, sa langue d'origine, le français, après un détour par ma langue d'origine, le russe.

JULIA HOLTER est enseignante à l'université catholique de l'Ouest et chercheuse associée à l'équipe «Multilinguisme, Traduction, Création» de l'ITEM. Elle a traduit les poètes russes Anna Glazova et Vladimir Aristov pour la collection de poésie russe contemporaine qu'elle dirige aux Éditions Joca Seria. Elle vient de publier Le Clair-obscur «extrême contemporain» : Pierre Bergounioux, Pierre Michon, Patrick Modiano et Pascal Quignard aux Éditions Rodopi/Brill, 2017. 


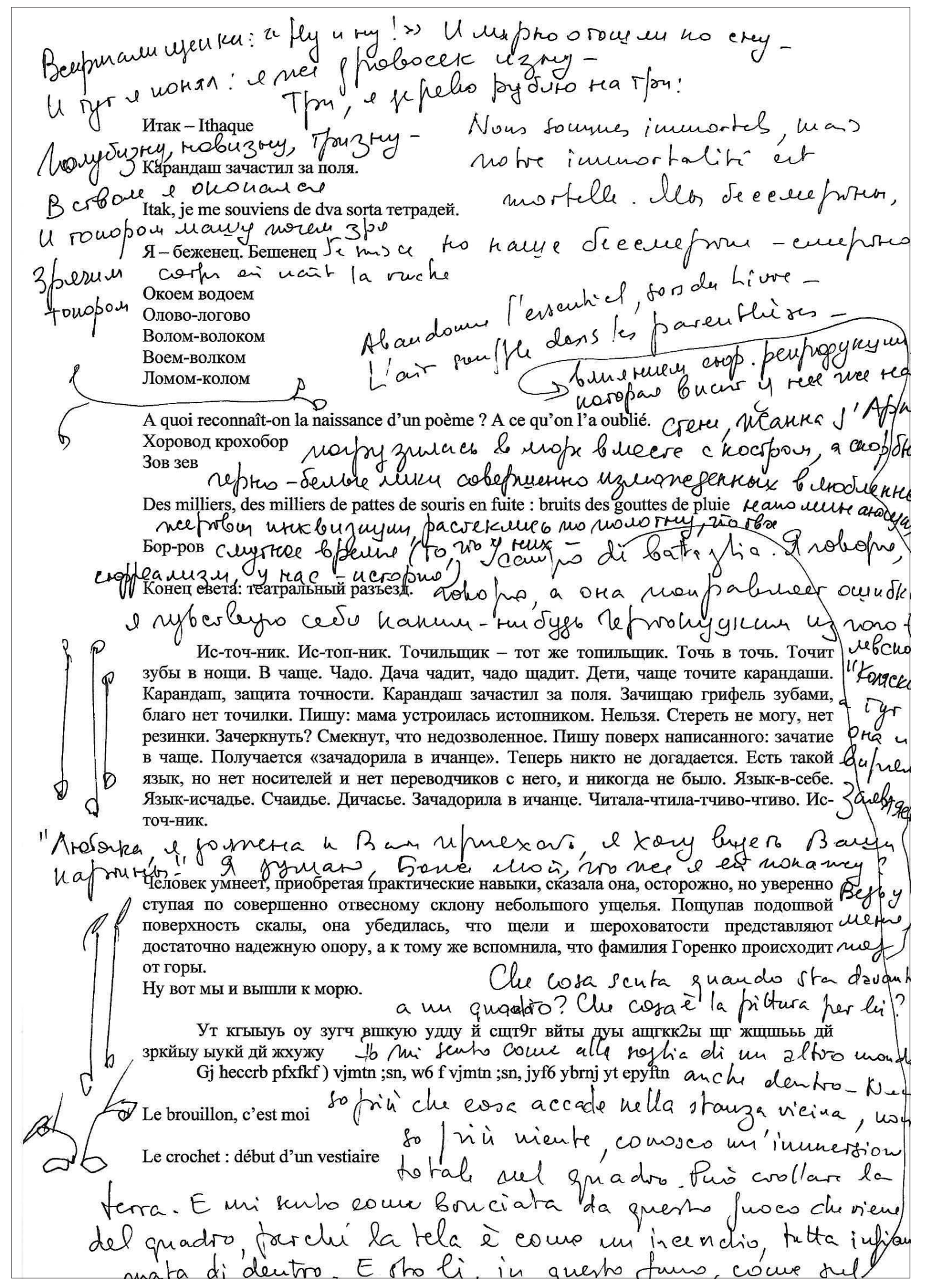

Fig. 1 : Marges 1 et Marges 4, en cours d'écriture, 2015, archives personnelles de Luba Jurgenson 


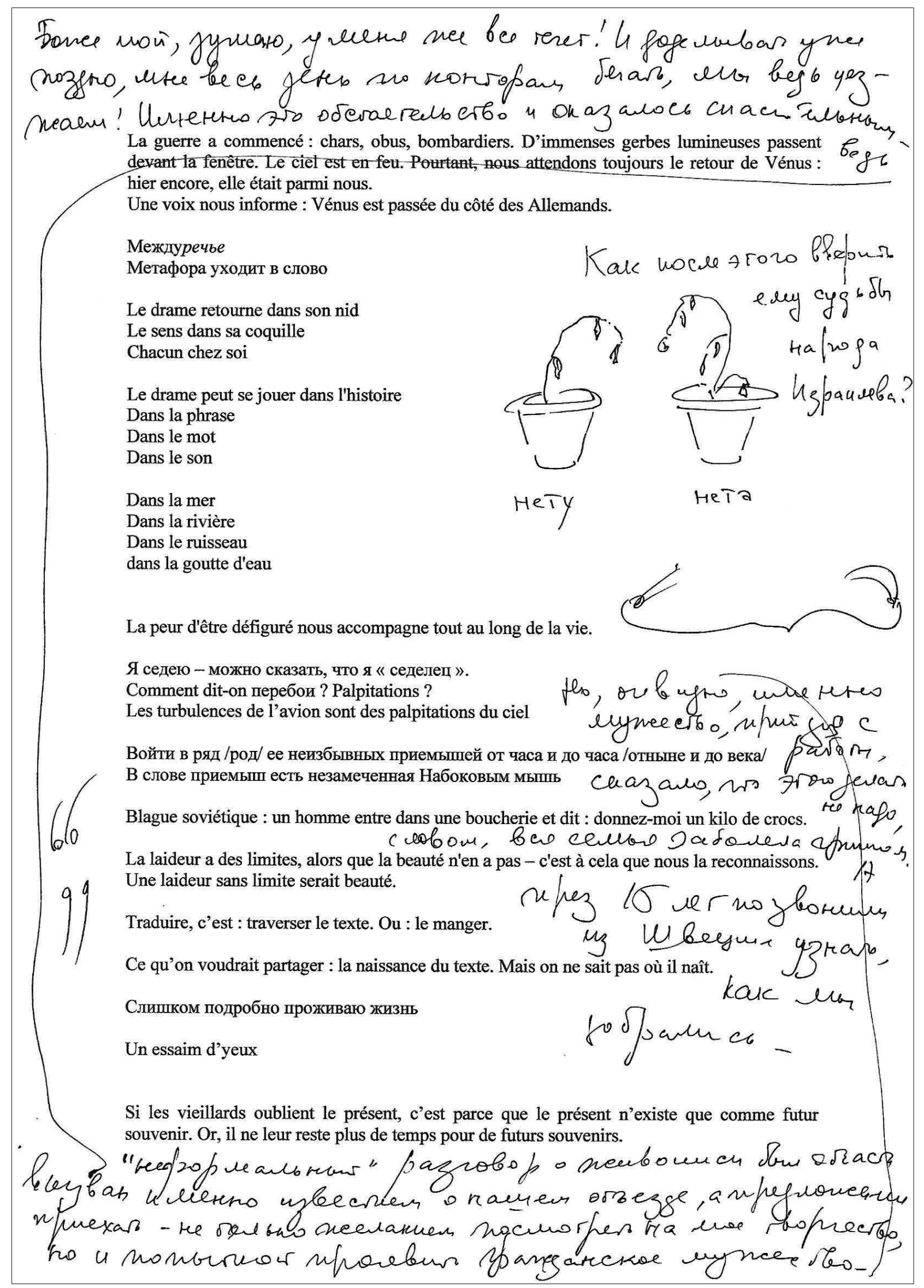

Fig. 2 : Marges 1 et Marges 4, en cours d'écriture, 2015, archives personnelles de Luba Jurgenson 